Merrell, K. W., Anderson, K. E., \& Michael, K. D. (1997). Convergent validity of the Internalizing Symptoms Scale for Children with three self-report measures of internalizing problems. Journal of Psychoeducational Assessment, 15:1, pp. 56-66. March 1997. (ISSN: 1557-5144 ) Version of record published by SAGE DOI: 10.1177/073428299701500105. This version may not be exactly the same as the version of record.

\title{
Convergent Validity of the Internalizing Symptoms Scale for Children with Three Self-Report Measures of Internalizing Problems
}

\author{
Kenneth W. Merrell, Kathryn E. Anderson, and Kurt D. Michael
}

\begin{abstract}
Data that serve to support the convergent validity of the Internalizing Symptoms Scale for Children (ISSC), a new child self-report measure for assessing internalizing symptoms, are presented. The results of three studies are presented, wherein ISSC scores were correlated with three established internalizing comparison measures: the Youth Self-Report, the Children's Depression Inventory, and the Revised Children's Manifest Anxiety Scale. Correlations were in the desired direction and magnitude for demonstrating the convergent validity of the ISSC as a self-report measure of internalizing symptoms of children. Strong correlations were found between the ISSC and the Internalizing broad-band score of the Youth Self-Report, whereas moderate to moderately high correlations were found between the scores of the ISSC and those of the Children's Depression Inventory and the Revised Children's Manifest Anxiety Scale. The ISSC appears to measure the internalizing construct in children and to have promise as a research and clinical tool for use with children ages 8 to 12 . Limitations of this investigation and implications for future research in this area are discussed.
\end{abstract}

\begin{abstract}
ARTICLE
In an effort to create empirical classification systems, several researchers have categorized children's emotional and behavioral problems into two dimensions: internalizing and externalizing disorders (Achenbach, 1985; Cicchetti \& Toth, 1991). Internalizing disorders, which include problems such as depression, anxiety, social withdrawal, and somatic complaints, have been broadly defined as inner-directed or over-controlled problems (Merrell, 1994). Externalizing disorders, on the other hand, have been described as outer-directed or under-controlled problems, including aggression, impulsivity, hyperactivity,
\end{abstract}


delinquency, and other overt behavioral problems (Reynolds, 1990).

Although the prevalence estimates of internalizing disorders in children vary depending on the particular disorder under investigation and the inclusionary criteria being used, even the lowest estimates create cause for concern. For example, Reynolds (1992a) suggested that the prevalence rates for childhood depression range from $4 \%$ to $12 \%$. Furthermore, prevalence estimates for childhood anxiety disorders have ranged from 3.5\% (Anderson, Williams, McGee, \& Silva, 1987) to $8.9 \%$ (Costello, 1989). It has been suggested that the existence of internalizing disorders in children may have a negative influence items and various personality/adaptive behavior items and scales. However, like the YSR, the BASC, despite its many positive characteristics, was not developed specifically for the purpose of assessing the broad spectrum of internalizing symptoms, Thus, both of these self-report measures may not include content usually not considered to be a main component of internalizing problems but certainly central to them, such as particular expressions of affectivity (see Stark, Kaslow, \& Laurent, 1993; Watson, Clark, \& Carey, 1988). In sum, although there are a few psychometrically sound children's self-report instruments designed to measure some aspect of internalizing problems, there is a continuing need for additional instrumentation in this area designed to meet the needs of clinicians and researchers who are specifically interested in investigating the broad spectrum of internalizing problems.

Given the need to develop and validate additional assessment tools in this area, the purpose of this research was to provide validity evidence for the Internalizing Symptoms Scale for Children (ISSC; Merrell \& Walters, 1996), a new instrument designed for the self-report assessment of internalizing symp toms of children ages 8 to 12 . The specific focus of this research was on the convergent validity of the ISSC. According to Gregory (1996), convergent validity of an instrument is evidenced when it is found to correlate highly with other established instruments measuring the same construct (or similar related constructs). This form of construct validity was investigated for the ISSC by making correlational comparisons of ISSC scores and scores of three established child self-report measures of internalizing problems.

\section{METHOD}

\section{Subjects}

Participants for this investigation included 256 mid-elementary-grade students (grades 3 through 6, ages 8 to 12) from two public school districts (one urban, one rural) The overall gender breakdown of participants was 119 boys (46\%) and 137 girls (54\%). Because the method of data collection approved for use in this investigation was blind to demographic characteristics of the subjects beyond grade, age, and gender, specific information on race/ethnicity, 
socioeconomic status, or special education classification of the subjects was not obtained. However, the racial/ethnic breakdown of the populations from which the samples were drawn (the two school districts) was approximately $85 \%$ Caucasian and $15 \%$ members of ethnic minority groups, almost entirely Hispanic, and, by inference, the ethnic/racial breakdown of the sample is considered to be roughly equivalent to these figures. Similarly, approximately $35 \%$ of the population from which the samples were drawn qualified for free or reduced-price lunch based on low family income status. Additionally, approximately $12 \%$ of the population from which the samples were drawn received special education services, primarily within inclusive regular education settings. Because this investigation included three separate groups of children who completed the ISSC and one comparison measure, the participants were essentially divided into three subsamples. The first subsample (in which participants completed the ISSC and the YSR) consisted of 51 participants (22 boys and 29 girls) who were in the common age range (11 to 12 years) for the two measures; all of the participants were in grade 6 . The second subsample (in which participants completed the ISSC and the CDI) consisted of 102 participants (47 boys and 55 girls) in grades 3 through 5 . The third subsample (in which participants completed the ISSC and the RC ) consisted of 103 participants (50 boys and 59 girls) in grades 3 through 5 .

\section{Instrumentation}

Internalizing Symptoms for Children. The ISSC is a self-report measure for use in screening for internalizing symptoms of children in grades 3 through 6 . The initial development of the ISSC was driven by the high degree of overlap among various internalizing symptoms and disorders (e.g., Brady \& Kendall, 1992; Reynolds, 1992b), coupled with the fact that most of the self-report measures for internalizing symptoms in children tend to be aimed at a specific syndrome (e-g., depression or anxiety) rather than at the larger construct of interrelated internalizing symptoms (Merrell, 1994). Thus, the ISSC was developed to provide a comprehensive self-report instrument for intermediate elementary age children to assess the symptoms of various internalizing problems (e.g., depression, anxiety, social withdrawal, somatic complaints) in a combined manner.

The ISSC includes 48 items that reflect either the presence or absence of a wide variety of internalizing symptoms. Children respond to each item by selecting one of the following statements that most accurately reflects how true they consider the statement to be for them: never true, hardly true, sometimes true and often true. Completion of the ISSC protocol takes most children an average of 15 to 18 minutes. About two thirds of the items are worded in a manner that suggests the presence of a particular internalizing characteristic, while about one-third of the items are presented in a manner that indicates either the absence of a specific internalizing symptom or the presence of positive affect or cognitions incompatible with specific internalizing symptoms. Item 
responses are keyed to a 4point scale (ranging from 0 to 3), where higher values always indicate greater internalizing symptoms.

Extensive and methodologically rigorous procedures were utilized during the development of the ISSC items to ensure a strong representation of the domain of children's internalizing symptoms and, thus, content validity of the instrument. For example, item development and selection procedures were based on current theories and empirical evidence regarding the internalizing dimension of child psychopathology (e.g., Cicchetti \& Toth, 199 1; Quay, 1986; Quay \& La Greca, 1986), involved exhaustive literature search procedures, and utilized a panel of experts to determine appropriateness and content validity of items. Development procedures and content validity evidence of the ISSC are described in more detail in the ISSC technical manual (Merrell \& Walters, 1996) and in other sources (Merrell, Crowley, \& Walters, in press; Walters \& Merrell, 1995).

Factor analytic studies have indicated that the ISSC includes two factors. The first factor, Negative Affect/General Distress, contains items that indicate the presence of specific internalizing symptoms or emotional distress. The second factor, Positive Affect, contains items that indicate the absence of internalizing symptoms/emotional distress or the presence of positive affect and cognitions incompatible with emotional distress. These factor analytic findings are compatible with those of previous researchers (e.g., Clark, Beck, \& Stewart, 1990; Watson et al., 1988) who have found that positive and negative expressions of affectivity are separate components that make unique contributions to the development or prevention of internalizing disorders such as depression and anxiety; they are not merely polar opposites along a continuum (Ingram \& Malcarne, 1995).

Based on analyses with the standardization sample of over 2,200 cases, the ISSC has an internal consistency reliability (coefficient alpha) of .92, with the two subscales having internal consistency reliabilities of .90 and .86, respectively. The test-retest reliability of the ISSC has been researched at short and medium time intervals and is quite strong. The mean reliability coefficient for 2-week retest intervals is .86 for the ISSC Total score and .85 and .81 for the two factor scores. ISSC reliability coefficients for 4 and 12-week retest intervals have been documented at .76 and .74 for the Total score and .73/.70 and $.79 / .72$ for the two factor scores (Michael, 1996).

The construct validity of the ISSC as a measure of internalizing symptoms has been demonstrated through three studies examining the sensitivity of the instrument in detecting important differences among various groups of children. Merrell and Dobmeyer (1996) investigated gender differences in ISSC score patterns and found a significant gender effect, with girls endorsing significantly more internalizing symptoms as a group than boys, an expected pattern based on the literature in this area. Sanders (1996) investigated differences in self-reported internalizing symptoms between seriously emotionally 
disturbed (SED) and regular education students and found that the SED students reported significantly higher levels of distress, another expected pattern of score distributions based on the large body of literature on social-emotional characteristics of SED students. Merrell, Gill, McFarland, and McFarland (1996) contrasted ISSC scores of students identified as talented and gifted with those of typical nongifted students and found that the gifted students reported significantly lower levels of internalizing symptoms. Although the body of evidence regarding social-emotional characteristics of gifted students is not as large as the previous two areas mentioned (and is sometimes contradictory), the direction of the findings in this particular study is also empirically supported by previous literature. Each of these three studies found internalizing patterns of critical item endorsement in the separation of groups through the use of discriminant function analysis. Other validity and clinical utility studies using the ISSC are ongoing. Sample ISSC items are presented in Table 1.

Table 1

Sample ISSC Items, Listed by Factor

\begin{tabular}{ll}
\hline Factor 1: Negative Affect/General Distress & Factor 2: Positive Affect \\
\hline I am shy. & I feel cheerful. \\
I worry about things. & I feel important. \\
I have bad dreams. & I have lots of energy. \\
I worry that I will hurt someone. & I do things as well as other kids. \\
I have trouble sleeping. & I like the way I look. \\
Lots of things scare me. & I do well in school. \\
When there is a problem, it is my fault. & I feel happy. \\
It is hard for me to breathe. & I like myself. \\
\hline
\end{tabular}

Comparison instruments. For the purpose of establishing convergent validity, three comparison instruments were utilized in separate correlational studies with the ISC: the Youth Self-Report (YSR), the Children's Depression Inventory (CDI), and the Revised Children's Manifest Anxiety Scale (RCMAS). These three comparison measures were selected because they represent both the general domain of internalizing symptoms (the YSR) and specific manifestations of types of internalizing symptoms (the CDI and RCMAS). These comparison measures were also selected because they are the most widely researched measures of their specific constructs in the child and adolescent population available.

It was hypothesized that, to demonstrate adequate validity as a measure of the general internalizing construct, the ISSC would need to have a strong relationship with the YSR (the other measure of the broad internalizing construct) and moderate relationships with the CDI and RCMAS, given that these measures are purported to measure specific aspects of the internalizing domain rather than the broad-band of internalizing symptoms. These comparison instruments and their psychometric properties are discussed briefly in this section. 
The YSR is a 119 item measure designed to assess the perception of 11- to 18year-old individuals regarding their own competencies and problems across situations. The 119 items are descriptive statements rated on a 3-point scale (not true to very true). Of the 119 items, 103 are statements about various problem areas, while the remaining 16 reflect socially desirable items endorsed by most subjects. Psychometric properties of the YSR from the standardization sample $(\mathrm{N}=1,315)$ include high internal consistency reliability $(\mathrm{r}=.83$ to 37$)$ and some evidence of validity. Although additional evidence supporting the validity of the YSR is needed, it appears to have the potential for being a useful selfreport instrument in a multiaxial assessment design. For this investigation, only the common items from the YSR Internalizing broad-band domain were used. Additionally, because the YSR spans a greater age range (11 to 18 years) than the ISSC (8 to 12 years), only participants in the common age range ( 11 to 12 years) were used in the comparison of the two measures.

The CDI is a 27-item self-report measure suitable for quantifying a range of depressive symptoms among school-age children and adolescents (6 to 17 years). It was originally developed as a downward age extension of the Beck Depression Inventory. The CDI item format consists of three statements about a particular depressive symptom of which the subject selects the statement that best summarizes his or her feelings for the past 2 weeks. The descriptive statements are rated on a 3-point scale, with higher scores indicating increasing severity in depressive symptoms. The 27 items provide information on five subscales (i.e., Negative Mood, Interpersonal Problems, Ineffectiveness, Anhedonia, and Negative Self-Esteem) and a total score on the individual's present depressive symptomology. Because the CDI is the most widely researched child self-report measure of the internalizing domain (Merrell, 1994), having been used in over 300 published studies, its psychometric properties are well documented. The CDI has been shown to have adequate to strong reliability and to have ample evidence of various types of validity.

The RCMAS is a 37-item self-report instrument designed to assess the level of trait anxiety (the tendency to be anxious across settings and times) in children and adolescents (6 to 17 years). The 37 statements are responded to in a Yes/No manner and provide information on three subscales (Physiological Anxiety, Worry and Oversensitivity, and Concentration Anxiety) and a Lie scale. The Lie scale consists of 9 items that are socially desirable but almost never true (e.g., "I am always nice to everyone."). A normative sample of nearly 5,000 cases was utilized in establishing the reliability and validity of this instrument. The RCMAS is also a widely researched measure, having been used in nearly 100 published investigations, and is considered to have adequate levels of reliability and validity. The RCMAS has been found to correlate highly ( $\mathrm{r}=.85$ ) with the Trait scale of the State-Trait Anxiety Inventory Scale for Children (STAIC) and to evidence low correlations $(r=.24)$ with the State scale of the STAIC, thus indicating that the RCMAS is best considered a measure of trait anxiety. 


\section{Procedure}

Participants were selected from regular classroom rolls. Signed consent forms were obtained from a parent or guardian of each participant prior to administration of the research instruments. Approximately $15 \%$ of the original pool of potential participants were not included in the study because their parent or guardian did not consent. The research instruments were group administered to the participants within regular classroom settings.

\section{RESULTS AND DISCUSSION}

Convergent validity coefficients for this investigation were obtained by computing Pearson product-moment correlations between the ISSC scores and the comparison instrument scores for each of the three studies. Correlations between the three ISSC scores and the Internalizing broad-band score of the YSR were as follows: ISSC Factor 1, .85; ISSC Factor 2.71; and ISSC Total score, .86 . These coefficients are high and indicate that the two instruments are measuring the same general construct, particularly when the correlations between the two total scores are considered. The $\mathrm{r}^{2}$ (coefficient of determination) value of the correlation between the two total scores (.86) is .74, indicating that $74 \%$ of the variability in each measure is attributable to variability in the other measure. Because it expresses the percentage of predictable variability between the two sets of scores, the $r^{2}$ statistic is considered to be an important measure of correlation in terms of the practical meaning or significance of relationships (Howell, 1982).

The validity coefficients for the study comparing the ISSC and the CDI are presented in Table 2. The correlations between the ISSC Total score and the CDI scores ranged from .46 to .70 for CDI subscale scores and .75 for the CDI Total score. Correlations between ISSC Factor 1 and the CDI subscales ranged from .74 to .62 , and a .60 correlation was obtained between Factor 1 and the CDI Total score. The correlations between ISSC Factor 2 and the CDI subscales ranged from .37 to .58 , with a .76 correlation between ISSC Factor 2 and the CDI Total score. Overall, the correlations between the ISSC and the CDI could be characterized as moderate to strong, and the two instruments appear to measure strongly related but slightly different constructs. The $\mathrm{r}^{2}$ value of the correlation between the total scores of the two measures is -58, indicating $58 \%$ shared variance. The .55 correlation between the ISSC Positive Affect score and the CDI Negative Mood score may be confusing, because one would presume such constructs to be negatively correlated. It must be remembered that when scoring the ISSC all items are coded so that higher values are always assigned to higher reports of emotional distress. Thus, this relationship is actually negative. 
Table 2

Convergent Validity of the ISSC and the Children's Depression Inventory (CDI): Correlations between ISSC Raw Scores and CDI T Scores

\begin{tabular}{lcccc}
\hline & \multicolumn{3}{c}{ ISSC Scores } \\
\cline { 2 - 4 } CDI Scores & $\begin{array}{c}\text { ISSC Factor 1: } \\
\text { Negative Affect/ } \\
\text { General Distress }\end{array}$ & $\begin{array}{c}\text { ISSC Factor 2: } \\
\text { Positive Affect }\end{array}$ & ISSC Total score \\
\hline Scale A: Negative Mood & .58 & .55 & .65 \\
Scale B: Interpersonal Problems & .42 & .37 & .46 \\
Scale C: Ineffectiveness & .46 & .58 & .56 \\
Scale D: Anhedonia & .62 & .56 & .70 \\
Scale E: Negative Self-Esteem & .43 & .52 & .52 \\
Total score & .60 & .76 & .75 \\
\hline
\end{tabular}

Note.-All correlations are significant at $p<.001$.

The validity coefficients for the study comparing the ISSC and RCMAS are presented in Table 3. The correlations between the ISSC Total score and the RCMAS scores ranged from .55 to .68 with the RCMAS subscales scores, and the total scores of the two measures were found to correlate at .78. Correlations between ISSC Factor 1 and the RCMAS subscales ranged from .55 to .69 , and a .79 correlation was obtained between Factor 1 and the RCMAS Total score. The correlations between ISSC Factor 2 and the RCMAS subscales ranged from .44 to .62 , with a .56 correlation between ISSC Factor 2 and the RCMAS Total score. As was true in the case of the relationship between the ISSC and CDI scores, the correlations between the ISSC and the RCMAS could be characterized as moderate to strong, and these two instruments also appear to measure strongly related but slightly different constructs, with the total scores sharing $61 \%$ of their variance $\left(r^{2}=.61\right)$

Table 3

Convergent Validity of the ISSC and the Revised Children's Manifest Anxiety Scale (RCMAS): Correlations between ISSC Raw Scores and RCMAS 'T Scores

\begin{tabular}{lccc}
\hline & \multicolumn{3}{c}{ ISSC Scores } \\
\cline { 2 - 4 } & $\begin{array}{c}\text { ISSC Factor 1: } \\
\text { Negative Affect/ } \\
\text { RCMAS Scores }\end{array}$ & $\begin{array}{c}\text { ISSC Factor 2: } \\
\text { Positive Affect }\end{array}$ & ISSC Total score \\
\hline Scale I: Physiological Anxiety & .69 & .48 & .68 \\
Scale II: Worry/Oversensitivity & .55 & .44 & .55 \\
Scale III: Social Concerns/Concentration & .62 & .62 & .67 \\
Total score & .79 & .56 & .78 \\
\hline
\end{tabular}

Note.-All correlations are significant at $p<.001$.

The correlations between ISSC Factor 2 and the RCUAS subscales ranged from .44 to .62 , with a .56 correlation between ISSC Factor 2 and the R C W Total score. As was true in the case of the relationship between the ISSC and CDI scores, the correlations between the ISSC and the RCMAS could be characterized as moderate to strong, and these two instruments also appear to measure strongly related but slightly different cons truce, with the total scores sharing 
$61 \%$ of their variance $\left(r^{2}=.61\right)$

Table 3

Convergent Validity of the ISSC and the Revised Children's Manifest Ansiety Scale (RCMAS): Correlations between ISSC Raw Scores and RCMAS T Scores

$\begin{array}{lccc} & \text { ISSC Scores } \\ \text { ISSC Factor 1: } & & \\ \text { Negative Affect/ } & \text { ISSC Factor 2: } & \\ \text { RCMAS Scores } & \text { Ceneral Distress } & \text { Positive Affect } & \text { ISSC Total score } \\ \text { Scale I: Physiological Anxiety } & .69 & .48 & .68 \\ \text { Scale II: Worry/Oversensitivity } & .55 & .44 & .55 \\ \text { Scale II: Social Concerns/Concentration } & .62 & .62 & .67 \\ \text { Total score } & .79 & .56 & .78 \\ \text { Note.-All correlations are significant at } p<, 001 . & & \end{array}$

In sum, these results provide partial evidence for the construct validity of the ISSC as a measure of internalizing symptoms of children. The strongest relationship found in this investigation was between the ISSC Total score and the Internalizing broad-band score of the YSR $\left(r=.86, \mathrm{r}^{2}=74\right)$. The YSR is one of the only child self-report instruments currently available that purports to assess internalizing symptoms in general (i-e., through the Internalizing broad-band items) rather than specific internalizing syndromes, and the strong relationship between the total scores of these two instruments suggests that the YSR and ISSC are measuring a similar construct. Because the lower limit for using the YSR is age 11 , while the ISSC extends down to age 8, this new measure may provide a valuable addition to available self-report instrumentation with preadolescent and intermediate elementary-age children. The CDI and the RCMAS are purported to measure the symptoms of depression and anxiety, respectively. As such, scores from a global measure of internalizing symptoms of children such as the ISSC would be expected to have moderate to moderately strong relationships with these two measures, and the range of correlations between the ISSC and the CDI and RCMAS is certainly at this expected level.

Although additional research to validate the ISSC for specific purposes is needed, these results are promising and indicate that the ISSC may prove to be a valuable addition to the available self-report instrumentation for research and clinical use with children. The ISSC appears to have solid technical properties, was developed using modem state-of-the-art procedures, and has been developed and refined so that it is quite user-friendly for children. Despite these encouraging findings, certain limitations of this investigation should be mentioned. Although the sample sizes for the three studies were adequate, it should be noted that they were drawn from a general population rather than a clinical population. Thus, it is unclear to what extent the rela-

[Archivist's note: Original publisher's version missing content after next four lines of text. See below:] 
tionships found between the ISSC and the three comparison measures would generalize to a clinical sample of children. Perhaps the greatest limitation of this study is that only one method of assessment (i.e., child self-report) was utilized. Each primary type of assessment (e.g., self-report, objective direct obser-

Achenbach, T. M. (1985). Assessment and taxonomy of child and adolescent psychopathology. Beverly Hills, CA: Sage.

Achenbach, T. M. (1991), Manual for the Youth Self-Report and 1991 Profile Burlington, VT: University of Vermont, Department of Psychiatry.

Anderson, J. C., Williams, S., McGee, R. \& Silva, P. A. (1987). DSM-III disorders in preadolescent children. Archives of General Psychiatry, 44, 69-76.

Brady, E. U., \& Kendall, P. C. (1992). Comorbidity of anxiety and depression in children and adolescents. Psycholog. ical Bulletin, 111, 244-255.

Campbell, D. T., \& Fiske, D. W. (1959). Convergent and discriminant validation by the multitrait-multimethod matrix. Psychological Bulletin, 57, 81-105.

Cantwell, D. P., \& Baker, L. (1989). Stability and natural history of DSM-III childhood diagnoses. Journal of the American Academy of Child and Adolescent Psychiatry, 28, 691-700.

Cicchetti, D., \& Toth, S. L. (1991). A developmental perspective on internalizing and externalizing disorders. In D. Cicchetti \& S. L. Toth (Eds.), Internal. izing and externalizing expressions of dys. function (pp. 1-19). Hillsdale, NJ: Erlbaum.

Clark, D., Beck, A., \& Stewart, B. (1990). Cognitive specificity and positive-negative affectivity: Complementary or contradictory views of anxiety and depression? Journal of Abnormal Psychology, 99, 148-155.

Costello, E. J. (1989). Developments in child psychiatric epidemiology. Joumal of the American Academy of Child and Adolescent Psychiatry, 28, 836-841.

Goldstein, P., Paul, G. G., \& SanfilippoCohen, S. (1985). Depression and achievement in subgroups of children with learning disabilities. Journal of Applied Developmental Psychology, 6, 263-275.

Gregory, R. J. (1996). Psychological testing:
History, principles, and applications (2nd ed.). Boston: Allyn \& Bacon.

Howell, D. C. (1982). Statistical methods for psychology. Boston: Duxbury Press.

Ingram, R. E., \& Malcarne, V. (1995). Cognition in depression and anxiety: Same, different, or a little of both? In K. D. Craig \& K. S. Dobson (Eds.), Anxiety and depression in adults and children (pp. 37-56). Thousand Oaks, CA: Sage.

Kendall, P. C., \& Brady, E. U. (1995). Comorbidity in the anxiety disorders of childhood. In K. D. Craig \& K. S. Dobson (Eds.), Anxiety and depression in adults and children (pp. 3-36). Thousand Oaks, CA: Sage.

Kovacs, M. (1985). The natural history and course of depressive disorders in children. Psychiatric Annals, 15, 387-389.

Kovacs, M. (1992). The Children's Depression Inventory. North Tonawanda, NY: Multi-Health Systems.

La Greca, A. M. (1990). Issues and perspectives on the child assessment process. In A. M. La Greca (Ed.), Through the ges of the child (pp. 3-17). Boston: Allyn \& Bacon.

Martin, R. P. (1988). Assessment of personal. ity and behavior problems. New York: Guilford Press.

Merrell, K. W. (1994). Assessment of behavioral, social, and emotional problems: Direct and abjective methods for use with childnen and adolescents. New York: Longman.

Merrell, K. W., Crowley, S. L., \& Walters, A. S. (in press). Development and factor structure of a self-report measure for assessing internalizing symptoms of elementary-age children. Psychology in the Schools.

Merrell, K. W., \& Dobmeyer, A. C. (1996). An evaluation of gender differences in self-reported internalizing symptoms of elementary-age children. Journal of Psy. choeducational Assessment, 14, 196-207.

Merrell, K. W., Gill, S. J., McFarland, H., \& 
McFarland, T. (1996). Internalizing symptoms of gifted and non-gifted elementary-age students: A comparative validity study using the Internalizing Symptoms Scale for Children. Psycholog in the Schools, 33, 185-191.

Merrell, K. W., \& Walters, A. S. (1996). The Internalizing Symptoms Scale for Children: User's guide and technical manual. Logan, UT: Department of Psychology, Utah State University.

Michael, K. D. (1996). An investigation of the temporal stability of self-reported inter. nalizing symptoms of elementary-age chil dren. Unpublished master's thesis, Utah State University, Logan.

Nasby, W., \& Yando, R. (1982). Selective encoding and retrieval of affectively valent information: Two cognitive sequences of children's mood states. Joumal of Personality and School Psychology, 43, 1244-1253.

Quay, H. C. (1986). Classification. In H. C. Quay \& J. S. Werry (Eds.), Psycho pathological disorders of childhood (3rd ed., pp. 1-34). New York: Wiley.

Quay, H. C., \& La Greca, A. M. (1986). Disorders of anxiety, withdrawal, and dysphoria. In H. C. Quay \& J. S. Werry (Eds.). Psychopathological disorders of childhood (3rd ed., pp. 73-110). New York: Wiley.

Reynolds, C. R., \& Kamphaus, R. W. (1994). Behavioral Assessment System for Children. Circle Pines, MN: American Guidance Service.

Reynolds, C. R., \& Richmond, B. O. (1985), Revised Children's Manifest Anxiety Scale (RCMAS) Manual. Los Angeles, CA: Western Psychological Services.

Reynolds, W. M. (1989). Reynolds Child Depression Scale. Odessa, FL: Psychological Assessment Resources.

Reynolds, W. M. (1990). Introduction to the nature and study of internalizing disorders in children and adolescents. School Psychology Revieu, 2, 137-141.
Reynolds, W. M. (1992a). Depression in children and adolescents. In W. M. Reynolds (Eds.), Internalizing disorders in children and adolescents (pp. 149-253). New York: Wiley.

Reynolds, W. M. (1992b). The study of internalizing disorders in children and adolescents. In W. M. Reynolds (Ed.), Internalizing disorders in children and adolescents (pp. 1-18). New York: Wiley.

Sanders, D. E. (1996). Internalizing Symptoms Scale for Children: A validity study with urban, African-American, seriously emotionally disturbed and regular education students. Unpublished doctoral dissertation, James Madison University, Harrisonburg, VA.

Speilberger, C. D. (1973). State-Trait Anxiety Inventory for Children. Palo Alto, CA: Consulting Psychologists Press.

Stark, K. D., Kaslow, N. J., \& Laurent, J. (1993). The assessment of depression in children: Are we assessing depression or the broad construct of negative affectivity? Journal of Emotional and Behavioral Disonders, 1, 149-154.

Stroessner, S. M., \& Mackie, D. M. (1992). The impact of induced affect on the perception of variability in social groups. Personality and Social Psychology Bulletin; 18, 546-554

Walters, A. S., \& Merrell, K. W. (1995). Written versus oral administration of social-emotional self-report tests for children: Does method of execution make a difference? Psychology in the Schools, 32, 186-189.

Watson, D., Clark, L. A., \& Carey, G. (1988). Positive and negative affectivity and their relation to anxiety and depressive disorders. Journal of Abnormal Psychology, 97, 346-353. 